[CONTRIBUTION FROM THE JOHN HARRISON LABORATORY OF CHEMistry:]

\title{
THE ATOMIC WEIGHT OF TANTALUM.
}

By William H. Chapin and Edgar F. SMith.

Received July 22, 1911.

\section{Introduction.}

It is scarcely necessary to mention the older work of $H$. Rose and Marignac, not by any means because it lacks importance, but because it is so well known. The values for tantalum that stood upon our lists of atomic weights for many years were worked out by these men, especially the latter.

The value now standing upon the table is $\mathrm{I} 8 \mathrm{I}$. This was determined by F. Willy Hinrichsen and N. Sahlbom by oxidation of metallic tantalum to the pentoxide. Their series contains five determinations, having among them an extreme variation of $I .2$ units, the lowest value being I 80.59 and the highest $18 \mathrm{I} .77$.

There has recently been published in THIS JournaL, 32, I I I 7, an article on the "Atomic Weight of Tantalum" by C. W. Balke. He determined the constant by transposing pure tantalum pentachloride into the oxide. ${ }^{1}$ His method of preparing the pure materials was extremely good, as was also the process of conversion into oxide. The mean of his series of eight very concordant values is $18 \mathrm{I} .52$.

A year ago an attempt was made in this laboratory by W. K. VanHaagen to determin the atomic weight of tantalum by transposing the pentabromide into oxide. Difficulties experienced in getting this compound in a pure condition made the values high and discordant; but it was nevertheless thought to be possible to overcome these difficulties, and with this in mind the present investigation was undertaken.

\section{Preparation of Pure Materials.}

Tantalum Oxide.-It is scarcely necessary to give an extended description of the methods employed in preparing pure tantalum oxide. They were such as have been used over and over again in this laboratory, and have been proved to be perfectly reliable. The starting-out material was potassium fluotantalate. This was six times recrystallized with centrifugal drainage. The reagents used were carefully purified, and nothing ever came in contact with the salt but hard rubber and platinum.

The oxide was obtained from this fluotantalate by treatment with the best obtainable sulfuric acid, subsequent hydrolysis, and washing. The last traces of water were finally removed by long extended ignition.

Special care was taken throughout this investigation to exclude dust.

Bromine.-The bromine was purified by dissolving in calcium bromide solution, and then distilling in apparatus constructed entirely of glass.

1 Proc. Am. Phil. Soc., 43, 443. 
This process removed every objectionable substance, including chlorine, of course. A possible trace of iodine would not be removed, but it has already been shown in this laboratory that tantalum iodide cannot be produced by combination with the oxide, and even if it were possible, it could not exist in preserice of an excess of bromine.

At the time of the first distillation an excess of potassium permanganate was added to decompose any hydrobromic acid, and to remove water the product was shaken with concentrated sulfuric acid, and subsequently redistilled.

Carbon.- The method of preparing the pentabromide called for the bromination of the oxide in presence of pure sugar carbon. It had been found very difficult, if not impossible, to prepare sugar carbon absolutely free from traces of hydrocarbons, which upon bromination would of course cause the production of hydrobromic acid. Van Haagen believed that it was the presence of this that indirectly caused the high value. His theory was that some compound analogous to the columbium compound, $\mathrm{Cb}_{2} \mathrm{O}_{5} \times \mathrm{HCl}$ was formed. With this in mind, special care was taken to remove hydrocarbons, the carbon being placed in a large porcelain crucible packed in charcoal in a Hessian crucible, and heated in a furnace until, upon removal, the cover of the porcelain crucible was found to be fused on. A combustion of this carbon, however, showed the presence of about 0.02 per cent. of hydrogen, and a second heating did not reduce the amount. A further attempt to remove the last traces of hydrogen consisted in the bromination of the carbon in a Jena tube at a high temperature. Even this treatment served only to slightly lower the percentage.

To prove the truth or fallacy of Van Haagen's theory, tantalum oxide was heated in an atmosphere of hydrogen bromide for an hour. The result was entirely negative. However, the presence of hydrogen would certainly insure the formation of a trace of water during the bromination of the oxide, and this would as certainly cause the decomposition of a corresponding amount of the pentabromide. Just what the product would be was not known at the time, but it was thought that it might be an oxybromide, $\mathrm{TaOBr}_{3}$, although the authorities denied the possibility of producing an oxybromide of tantalum. Considering, then, the seeming impossibility of removing the last traces of hydrogen from the carbon, it looked as if the only alternative was to prepare the bromide with possibly a slight uncertainty as to its composition, and then rely upon methods of fractional distillation for obtaining an absolutely pure product.

Concerning the carbon, it remains only to be said that 20 grams, when burned completely in a porcelain crucible, left no ponderable residue. 


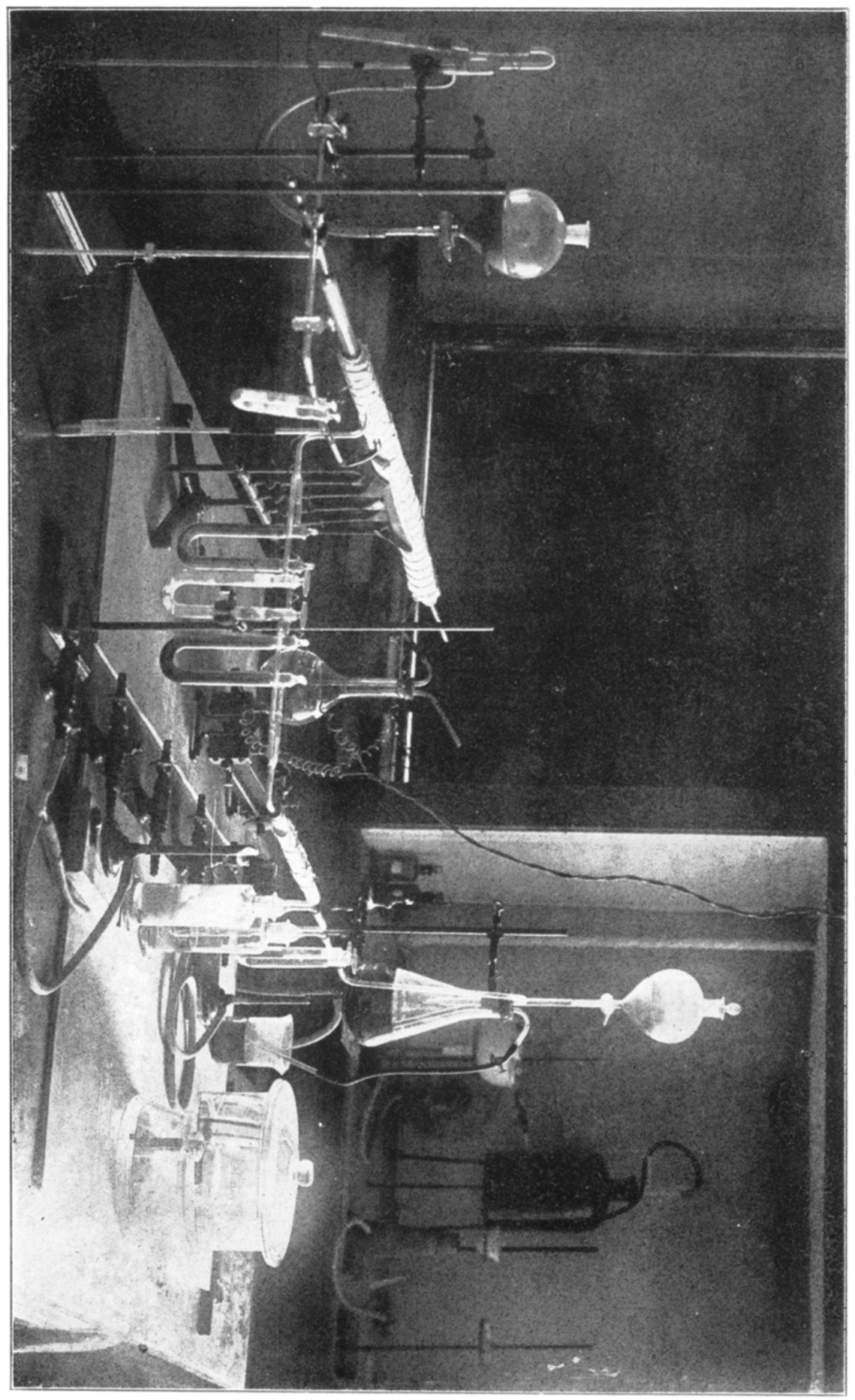





\section{Preparation of the Pure Bromide.}

Bromination of the Oxide. - The method of preparing a bromide by conducting bromine vapors over a red-hot mixture of an oxide and sugar carbon is familiar to almost every one. It needs only to be said that special care was taken in this case in the construction of the apparatus. All parts which were to come in contact with bromine were made entirely of glass, either sealed together or connected by ground joints. The bromine was carried by a current of carbon dioxide, special care being exercized to obtain this pure and absolutely dry. The cut of apparatus used later in the fractional distillation shows very well the arrangement of the parts used in this case. The carbon dioxide, after passing through sodium bicarbonate to remove traces of hydrochloric acid, and through sulfuric acid to remove water, passed next over a red-hot copper spiral to remove any oxygen, and then to the system of U-tubes seen in the foreground. Here the course was divided, the gas passing either through the bromine tube, and then a phosphorus pentoxide tube, or through the latter tube alone. This arrangement made it possible to clear the tube of air and subsequently of bromine with perfectly dry carbon dioxide. This gas was generated by action of dilute hydrochloric acid on marble. It scarcely needs mentioning that the latter was boiled under reduced pressure to remove air. The generator was so arranged that the spent liquid could be withdrawn without opening. In fact it was kept in operation for more than a month without the admission of any air. All the acid used was boiled and was transferred to the tap-funnel without contact with air. It was subsequently kept entirely in an atmosphere of carbon dioxide.

Distillation of the Pentabromide.-By the above-mentioned method about roo grams of the pentabromide were prepared. The only possible impurity that this could have contained was some compound of tantalum related to the pentabromide but containing an excess of oxide, and the distillation was conducted with the intent of removing this. It had previously been noticed that when the crude product was redistilled a much less volatil residue remained, and it was hoped that a few distillations would completely remove this. The apparatus used for the first distillations consisted simp'y of a Jena tube having two constrictions near one end. The charge (about 50 grams) was placed in the tube and a rapid current of carbon dioxide conducted for some time to remove air. Heat was then applied; when at a temperature of about $250^{\circ}$ the salt melted, and immediately began to distil away, condensing beyond the constriction in beautiful dark red laminae. After completing one distillation the heating apparatus was shifted, and the bromide distilled beyond the next constriction. Finally, after completing the distillation, the salt was melted down and allowed to cool to a hard mass. 
When entirely cool this contracted away from the glass, and could easily be dropped out in a single piece directly into a large weighing tube standing in a desiccator. Four distillations made after this manner left the bromide most beautiful in appearance, but the tube was not left entirely clean. A slight film of yellowish color was still seen adhering to the glass. At a high temperature, however, this was somewhat volatil, and it was therefore uncertain whether the separation was complete. A trial determination of the atomic weight made with this material gave the value I83.0O, hence it did not seem probable that the maximum of purity had been reached.

It was thought that if the bromide could be distilled a number of times without removal from the tube the desired end might be attained. For this purpose a Jena tube seven feet long having six constrictions was prepared, and the distillation conducted with this as before. The result was disappointing, for even the sixth distillation left the same yellowish film on the glass--thinner than before, perhaps, but distinctly visible. A trial determination of the atomic weight made at this point gave the value 182.70 -only a slight improvement over the first value.

With another tube of similar construction six more distillations were conducted, part of them in an atmosphere of bromine, but there was no apparent improvement, a slight film being left after each distillation. Two trial determinations made here gave the values 182.28 and 182.32 , indicating homogeneity of material, and something of a change in composition. However, it scarcely seemed as if pure material could ever be obtained by this method if sixteen distillations had apparently failed to give it. It was thought that possibly the carbon dioxide still contained traces of air (oxygen), or that the carbon dioxide itself brought about a slight decomposition of the bromide. At any rate it was decided to carry out the process of distillation in a vacuum.

Sublimation in Vacuo: The Oxybromide.-The apparatus used for the vacuum distillation is shown in the cut. The distilling tube was connected on the one hand with a mercury pump, which gave a vacuum of I $\mathrm{mm}$., and on the other with a carbon dioxide or nitrogen generator, an arrangement which permitted the alternate evacuation and filling of the tube. This was necessary, since it was found best to use a tube with several constrictions as before, and to seal off after each distillation so as to preclude the possibility of contaminating the pure material by an after-distillation of the separated impurity. The heating was accomplished by means of a resistance coil of "nicrome" ribbon. This was wound upon a glass jacket, and so arranged that one end of the tube could be heated to $300^{\circ}$ while the other remained at about $200^{\circ}$. The jacket. was made movable, and during a distillation was pushed over the inner tube step by step until the temperature was just sufficient to distil the 
pentabromide and leave the impurity behind. It should be noted here that in a complete vacuum the former does not melt but simply sublimes.

The tube seen in the cut extending downwards from the end of the combustion tube dips into a tube of mercury, and acts as a manometer. The stopcocks on either side were lubricated with a mixture of rubber and paraffin, making them absolutely air-tight. The rubber stopper at the end of the combustion tube was covered, after insertion, with several coats of lacquer. After evacuation the apparatus was always left for some time to be absolutely sure that there was no leakage, the manometer reading being compared with that of a barometer standing near by.

This method of fractionation did not always work out smoothly, but in general the results were very good. Using a tube with three constrictions, and sealing off after each distillation, the amounts of residue were about like those shown in the following example:

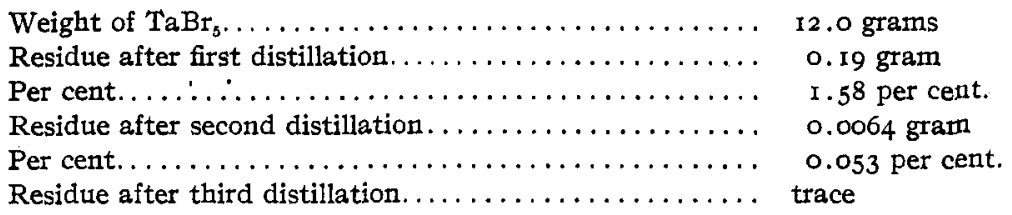

Considering these facts, it was believed that the pentabromide obtained by distillation in vacuo was pure. Trial determinations on different samples prepared in this manner gave values for the atomic weight ranging from 181.7 to 181.9 .

The nature of the yellow residue: As has been suggested, it seemed quite probable that, in spite of statements to the contrary, an oxybromide of tantalum did exist, and that this residue was that very compound. As obtained in these distillations, it was an extremely light, feathery substance, very deceptive in its appearance, leading one to believe that there was a much greater quantity than really existed. One analysis conducted on a very small sample gave the following results:

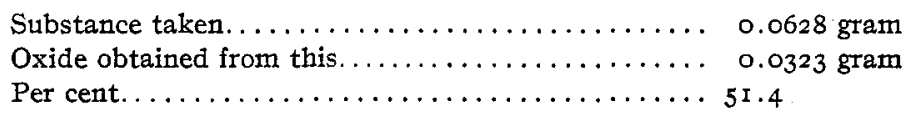

Allowing $\mathrm{I}_{2} 2$ as the atomic weight of tantalum, the oxybromide, $\mathrm{TaOBr}_{3}$, would give of oxide 50.7 per cent. Therefore, considering the smallness of the sample, the agreement seemed as close as could be expected, and there was left little reason to doubt that this was really a true oxybromide of tantalum.

\section{Determination of the Atomic Weight.}

The method of determining the atomic weight through the pentabromide is rather simple. This salt, like the chloride, is instantly decom- 
posed by water, halogen acid being given off and the metallic base being left behind in the form of the white hydrated oxide. The latter, upon strong ignition, goes to the pentoxide, $\mathrm{Ta}_{2} \mathrm{O}_{5}$. To bring about complete expulsion of bromine, nitric acid must be used in small amounts, this being added after the greater part of the hydrobromic acid has been evaporated off.

From the above it is very evident that up to the time when the pentabromide has been weighed out it must be kept absolutely out of contact with every trace of moisture. Indeed this fact constitutes the most difficult problem connected with the preparation and preservation of this substance. As has already been indicated, the pentabromide, after distillation and subsequent cooling, contracted sufficiently to separate itself from the walls of the combustion tube, and could therefore be dropped out in a single piece. If the container was placed in a large desiccator under phosphorus pentoxide, there was very little chance for decomposition. This, in fact, was the method pursued, and to make a safe transference of the bromide the end of the combustion tube was simply placed within the mouth of this container and then tilted until the piece slid out, or if necessary was pushed out with a glass rod. To further prevent any contact with moist air, the stopper of the container was very slightly lubricated with paraffin, and held in place by means of a rubber band. The piece of pentabromide as obtained usually weighed from 12 to 20 grams, and could easily be broken up into pieces of convenient size by tapping with a long glass rod, a process which required only a partial removal of the desiccator cover, and certainly could not have allowed any moisture to reach the salt. It might be stated here that the proof of this statement was seen later, when, upon running a series of four determinations and thus opening the tube four different times, the last of the series gave the lowest result, which would not have been the case had an appreciable amount of moisture reached the tube.

The weighing out of the sample of pentabromide for analysis was conducted in the following manner: A glass weighing tube was used whose stopper fitted over the outside of the neck. This prevented soiling at the time of emptying out the sample, and consequent trouble about getting the stopper back. This weighing tube was placed open in the desiccator beside the tube containing the pentabromide, and in its mouth was placed a wide short-stemmed funnel. At the same time the rubber band about the bromide tube was removed and the stopper slightly loosened. The caver of the desiccator was then replaced and left for half an hour, until every trace of moisture should be removed from the weighing tube. Next the desiccator was partially uncovered, and by means of a pair of long glass forceps a single piece of the pentabromide was quickly transferred to the weighing tube, the funnel removed, and 
the stopper dropped in place. Great care was taken not to push the latter on, since the contained air could thus be slightly compressed and would remain so. It was allowed to settle down slowly by its own weight, and finally gently tapped into place.

Having thus made the transference, the weighing tube was placed upon the balance pan and allowed to remain there for fifteen minutes, at the end of which time it was weighed. (The balance was one of Troemner's best make, and the weights were carefully calibrated.) After the weight was taken the sample was carefully dropped out into a weighed porcelain crucible, the latter standing inside a larger crucible of the same kind, and the tube was then reweighed. The greatest care was always taken to touch the tube as little as possible, and also to allow a sufficient time for readjustment after such contact. In the case of the crucibles a counterpoise of almost exactly the same size and weight was used. In applying the vacuum correction the value 4.67 was taken as the density of the pentabromide. This had previously been determined by Van Haagen. The correction amounted to 0.00013 gram for each gram of substance taken.

The crucible containing the pentabromide was placed on a water bath and about half filled with pure distilled water. With a solid piece of the pure pentabromide there was never any tendency to spatter or hiss. In fact the piece usually remained perfectly bright and apparently unaffected for several minutes after the water was applied. It should be noted here that the water bath was constructed entirely of porcelain, and only distilled water was ever used in it.

When the sample had finally become completely hydrolyzed the water and acid were evaporated off. To remove the last traces of bromine the oxide was at this point covered with pure Io per cent. nitric acid, and the evaporation repeated.

The crucible was next placed within a porcelain air bath, and with cover in place was heated cautiously until the oxide became apparently dry, and all the nitric acid was expelled.

The final step was the strong ignition, which was conducted first over a Bunsen burner and then over a good blast lamp. The blasting was, of course, repeated until constant weight had been reached, and usually required about forty-five minutes.

In weighing the oxide no vacuum correction was applied since the density of tantalum oxide is not far from that of the brass weights. In calculating the atomic weight the value 79.92 was taken for bromine; that of oxygen was 16 , of course. The following table contains the results of all determinations which were carried through to completion and were not known to be in error: 
Wt. of TaBrs.
(vac.).

0.86837

I. 50903

I. 56554

I . 23239

I. 3I 8 I 5

1. 31702

I. 20090

I. 04050
Wt. of $\mathrm{Ta}_{2} \mathrm{O}_{5}$.
(vac.).

0.33117

o. 57570

0.597 I 8

0.47030

0.50295

0.50244

0.45830

0.39688

University of PenNsylVania.

At. wt.

I 81.68

I 81.80

I $8 \mathrm{I} .75$

I $8 \mathrm{I} .9 \mathrm{I}$

I 8 I. 85

I 81.80

I8I. 9 I

r 81.74

Mean, I81.80

[CONTRIBUTION FROM THE JOHN HARRISON LABORATORY OF CHEMISTRY.]

\section{THE ACTION OF HYDROFLUORIC ACID GAS UPON CERTAIN OXIDES.}

By Walter K. Van haAgen and Edgar f. Smith,

Received July 22, 1911.

From time to time in this laboratory various metallic oxides have been exposed at varying temperatures to the influence of gaseous halogen acids. In the past summer we have taken occasion to ascertain the volatility or non-volatility of certain oxides when heated in hydrofluoric acid gas. The latter was generated from well dried calcium fluoride and concentrated sulfuric acid contained in a platinum retort which was connected with a platinum combustion tube, in which there was also a platinum boat carrying the material to be acted upon. The constricted portion of the combustion tube was cooled by means of a moist wad of filter paper, while its extreme end was allowed to dip into distilled water. The retort and its contents were gradually heated in a glycerol bath, by which means a constant and even flow of hydrofluoric acid gas was obtained.

On treating strongly ignited and powdered aluminium oxide as above indicated, the boat and its contents became exceedingly hot to the touch but there was no volatilization of the material. At a white heat a small portion of fluoride of aluminium could be detected in the receiver. The conversion of oxide into fluoride was perfectly quantitative after heating in the bath for twenty minutes.

Yttrium oxide suffered a complete change at a red heat to fluoride or oxyfluoride. There was no volatilization and, therefore, no yttrium was found in the receiver.

The conversion of lanthanum oxide to fluoride was also quantitative and there was no volatilization of lanthanum salt. Finely divided quartz volatilized easily and completely without the application of external heat. The heat of the reaction was intense. 